\title{
SCMA Receiver Design for Cellular Uplink Transmission Coexisted with D2D
}

\author{
Yukai Liu, Wen Chen, Fan Wei \\ Shanghai Institute of Advanced Com. and Data Sciences \\ Department of Electronic Engineering \\ Shanghai Jiao Tong University, Minhang 200240, China \\ Email: \{liuyukai,wenchen,weifan89\}@sjtu.edu.cn
}

\author{
Fabrice Labeau \\ Department of Electrical and Computer Engineering \\ McGill University, 3480 University Street \\ Montreal, QC. H3AoE9, Canada \\ Email: fabrice.labeau@mcgill.ca
}

\begin{abstract}
Sparse code multiple access (SCMA) is one of the most concerned non-orthogonal multiple access schemes in 5G communication system. Meanwhile, device-to-device (D2D) communication is also one of the key technologies for 5G network to improve the spectral efficiency. In cellular systems with SCMA, the non-orthogonal nature renders resource blocks support for more users so that an overloaded system can be achieved. To further improve the system capacity, a D2D and uplink cellular coexisting network is proposed in this paper. The interference between D2D and cellular users are handled. Since conventional message passing algorithm (MPA) has the exponential complexity, the receiver for cellular users is based on some low complexity methods and that for D2D users is also associated with this method. Simulation results show that the proposed hybrid network has an acceptable BER performance loss compared to the general uplink SCMA system, while the proposed SCMA receiver can reduce the decoding complexity significantly compared to the conventional MPA.

Index Terms-device-to-device communication, sparse code multiple access, hybrid network, low complexity decoder.
\end{abstract}

\section{INTRODUCTION}

The growing demand for higher data rates and larger capacity requires new communication designs for the next generation $(5 \mathrm{G})$ wireless networks. Some key technologies for 5G systems include device-to-device (D2D) communication, non-orthogonal multiple access techniques along with massive multiple-input multiple-output (MIMO), ultra-dense radio networking, all-spectrum access, and so on [1].

D2D communication is one of the promising technologies, which allows two nearby devices to communicate without base station (BS) or with limited BS involvement. As D2D communication belongs to class of cooperative communication, this technique has the benefits in terms of link reliability, spectral efficiency, system capacity, and transmission range [2]. When this technique is incorporated into cellular networks, the system capacity can be improved.

Among all the non-orthogonal multiple access techniques, sparse code multiple access (SCMA) has been proposed as a promising method. SCMA is introduced by Nikopour and Baligh [3]. In SCMA, coded bits are directly mapped to the multidimensional complex lattice point (called a codeword), and the codewords are designed to be sparse. Compared to other multiple access techniques, the sparsity of SCMA codewords enables massive connectivity and overloaded feature. Besides, it also renders the use of the suboptimal message passing algorithm (MPA) to solve the problem of multi-user detection. However, despite of the sparsity of SCMA, the decoding of codewords brings a high computational complexity. To solve this issue, several low complexity MPA designs are proposed [4]-[6]. The authors in [4] propose a modified MPA based on partial marginalization (PM). The authors in [5] design a residual-aided message propagation (R-aided MPA) to accelerate the convergence speed. In [6], a codebook is designed to reduce the number of projections in each orthogonal frequency division multiple access (OFDMA) tone.

Some work further considers D2D communication combined with SCMA [7]-[13], [19], [20]. They assume the cellular users and D2D pairs can share certain SCMA layers and some SCMA layers may be reused. In order to maximize the total rate of cellular users and D2D pairs, a coalition game based scheme is proposed [7]. Besides, opportunistic SCMA codebook allocation (OSCA) [8] and interferenceaware hypergraph based codebook allocation (IAHCA) [9], [19] are proposed to obtain the suboptimal solution and to reduce the cross-tier interference between cellular users and D2D users. In OSCA, codebooks are opportunistically assigned based on the channel conditions of cellular links and D2D links, while in IAHCA, hypergraph model is used to characterize the interference, and then each orthogonal SCMA resource is allowed to be shared by one cellular link and more than one D2D links. In [10], SCMA codebooks are not reused for all users, while D2D links reallocate the resources to cellular users in a non-orthogonal mode. By restricting the distance from D2D transmitter to the base station, the BER performance and the convergence behavior can be better. When a proportion of available resources are allocated to D2D users in a dedicated manner, both D2D reuse mode and D2D dedicated mode are considered [11],[12]. A graph-based joint mode selection and resource allocation algorithm with predefined threshold is proposed to maximize the system sum rate [11]. Besides, the work in [12] further considers other issues such as admission control, partner assignment, and power allocation. Moreover, a channel gain based mode selection criterion as well as a greedy-style partner assignment scheme are proposed. Another D2D underlaying cellular network makes use of a different hybrid multiple 
access technique [13]. To improve the performance of D2D users, SCMA is employed for cellular users while OFDMA is employed for D2D users. However, the interference still exists. Then, an interference graph based resource allocation scheme is proposed to minimize the total transmit power subject to a rate constraint.

This paper considers a D2D and uplink cellular hybrid network model to support users' communication simultaneously. Different from the above mentioned works [7]-[13], we focus on the receiver design for both cellular users and D2D users. We assume that cellular users employ SCMA. For uplink cellular users, conventional MPA needs modifications due to the interference of D2D communication. During the iterative process of MPA, the extrinsic information also considers the transmitted codewords of D2D users. More importantly, the large codebook size makes it hard to compute all Euclidean distances and also increases the computational complexity. Therefore, low complexity decoding methods are necessary for the receiver design of the proposed network. We utilize list sphere decoding (LSD) [14] based MPA in this paper so that the search space can be reduced. We also analyze the computational complexity for different decoding algorithms. For D2D users, we consider the ML criterion based optimal receiver with the interference of uplink cellular users first. Besides, the sphere decoder also reduces the interference space for the receiver design of D2D users.

The rest of the paper is organized as follows. Section II describes the network model and illustrates the related multiple access techniques. Besides, The SCMA basic structure as well as notations are also introduced. The receiver design for both cellular users and D2D users are proposed in section III. The conventional MPA algorithm and the LSD based decoding algorithm are introduced for cellular users, along with the complexity analysis. We also illustrate the decoder for D2D users. Section IV shows the numerical results. The paper finally concludes in section V.

\section{System ModeL}

\section{A. The D2D and Cellular Hybrid Networks}

Fig. 1 shows a D2D and uplink cellular hybrid network. In this network, we consider $J$ cellular users and $J_{d 2 d}$ D2D pairs. The $i$ th cellular user is denoted as $C_{i}$, and the D2D transmitter and receiver of the $i$ th D2D pair are denoted as $D T_{i}$ and $D R_{i}$, respectively.

For general uplink communication system, SCMA can be applied. Cellular users are allocated with SCMA codewords spread over OFDMA tones. Further, this network contains D2D communication. Each D2D receiver only concerns about its own information. Thus, OFDMA is more reasonable for D2D users.

\section{B. SCMA Structure}

The SCMA encoder can be defined as a mapping from $\log _{2}(M)$ coded bits to a $K$-dimensional complex codebook of size $M$. We select $N$ dimensions from $K$ for the sparse

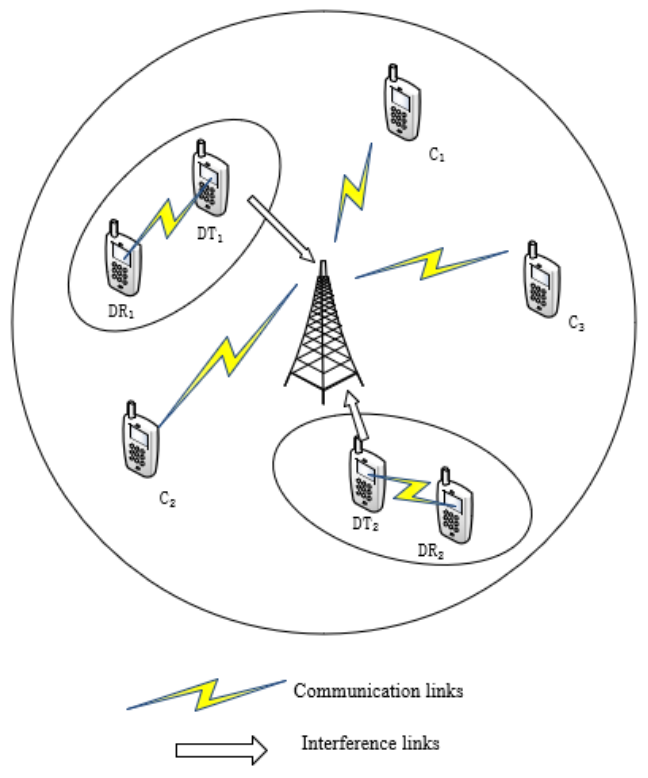

Fig. 1: D2D and cellular hybrid network.

codebook design, in other words, each $K$-dimensional complex codeword are only non-zero in $N$ dimensions. Thus, $J$ cellular users are allocated with $K$ OFDMA subcarriers. In this model, we consider the case $J_{d 2 d}<K$ in order to restrict the interference range between cellular users and D2D users.

\section{Notations}

Throughout this paper, the following notations will be used. The lowercase letter $x$ denotes a scalar, the bold lowercase letter $\boldsymbol{x}$ denotes a column vector, and a matrix is represented by a bold uppercase letter $\boldsymbol{X}$. The superscript $(\cdot)^{T}$ denotes matrix transpose and $(\cdot)^{\dagger}$ denotes conjugate matrix transpose. $\operatorname{diag}(\boldsymbol{x})$ denotes a diagonal matrix with the diagonal entries being vector $\boldsymbol{x}$. Besides, $\xi \backslash j$ denotes the set $\xi$ in which the element $j$ is excluded. $\boldsymbol{I}$ denotes the identity matrix. $\mathbf{0}$ and $\boldsymbol{O}$ denote a column vector and a matrix with all zero elements, respectively.

\section{ReCEIVER Design for CEllular AND D2D}

\section{A. MPA Decoder for Cellular Users}

The received signal $\boldsymbol{y}$ after synchronous layer multiplexing at base station can be expressed as

$$
\boldsymbol{y}=\sum_{i=1}^{J} \operatorname{diag}\left(\boldsymbol{h}_{i}\right) \boldsymbol{x}_{i}+\sum_{j=1}^{J_{d 2 d}} \operatorname{diag}\left(\boldsymbol{h}_{j}^{\prime}\right) \boldsymbol{x}_{j}^{\prime}+\boldsymbol{n},
$$

where $\boldsymbol{h}_{i}$ and $\boldsymbol{x}_{i}$ are the channel vector and SCMA codeword vector of cellular user $i, \boldsymbol{h}_{j}^{\prime}$ and $\boldsymbol{x}_{j}^{\prime}$ are the channel vector and D2D codeword vector of D2D transmitter $j, \boldsymbol{n}$ is the additive Gaussian noise vector with mean $\mathbf{0}$ and covariance matrix $\sigma_{n}^{2} \boldsymbol{I}$. Besides, we define that $\boldsymbol{x}=\left[\boldsymbol{x}_{1}^{T}, \boldsymbol{x}_{2}^{T}, \ldots, \boldsymbol{x}_{J}^{T}\right]^{T}$ and $\boldsymbol{x}^{\prime}=\left[\left(\boldsymbol{x}_{1}^{\prime}\right)^{T},\left(\boldsymbol{x}_{2}^{\prime}\right)^{T}, \ldots,\left(\boldsymbol{x}_{J_{d 2 d}}^{\prime}\right)^{T}\right]^{T}$. For base station, the information of D2D users are insignificant, so D2D codewords could be regarded as interference. 
SCMA systems utilize MPA for sake of the sparsity of SCMA codewords. Considering $M$ constellation points are uniformly distributed, cellular users' codewords can be estimated based on ML criterion

$$
\hat{\boldsymbol{x}}=\arg \max _{\boldsymbol{x} \subset X}\{M(\boldsymbol{x})\},
$$

where

$$
M(\boldsymbol{x}) \propto \sum_{\boldsymbol{a} \subset X_{d 2 d}} p\left(\boldsymbol{x}^{\prime}=\boldsymbol{a}\right) \exp \{F(\boldsymbol{a}, \boldsymbol{x})\},
$$

where $X_{d 2 d}$ means the set of D2D transmitted codewords, $p(\cdot)$ means the probability value for each possible D2D transmitted codeword. Besides, we have

$$
F(\boldsymbol{a}, \boldsymbol{x})=-\frac{1}{\sigma_{n}^{2}}\left\|\left(\boldsymbol{y}-\boldsymbol{H}^{\prime} \boldsymbol{a}\right)-\boldsymbol{H} \boldsymbol{x}\right\|^{2},
$$

where $\boldsymbol{H}=\operatorname{diag}\left\{\boldsymbol{h}_{1}^{T}, \boldsymbol{h}_{2}^{T}, \ldots, \boldsymbol{h}_{J}^{T}\right\}$ is a block diagonal matrix as well as $\boldsymbol{H}^{\prime}=\operatorname{diag}\left\{\left(\boldsymbol{h}_{1}^{\prime}\right)^{T},\left(\boldsymbol{h}_{2}^{\prime}\right)^{T}, \ldots,\left(\boldsymbol{h}_{J_{d 2 d}}^{\prime}\right)^{T}\right\}$.

The form in (4) is more complicated than general SCMA system due to the interference of D2D users. For base station, the information of D2D users are absolutely unknown so we must compute the conditional probability to eliminate the influence of D2D users.

SCMA structure can be formulated as a factor graph which is shown in Fig. 2, where some resource nodes are also occupied by D2D users in proposed network model.

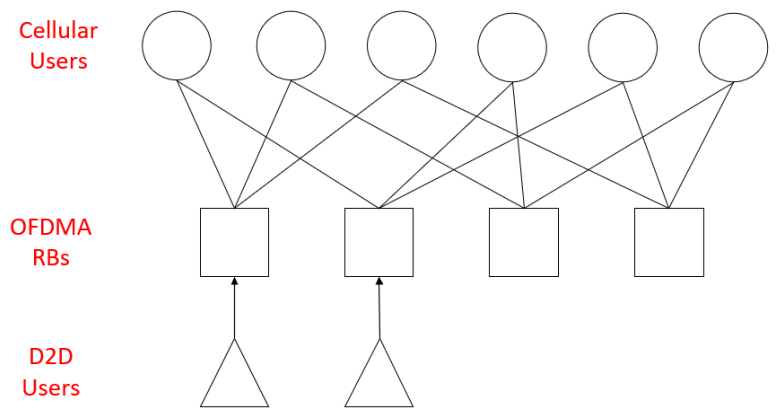

Fig. 2: Factor graph with $J=6, K=4, N=2$, and $J_{d 2 d}=2$.

Besides, the corresponding SCMA indicator matrix is shown as follows

$$
\boldsymbol{F}=\left[\begin{array}{llllll}
1 & 1 & 1 & 0 & 0 & 0 \\
1 & 0 & 0 & 1 & 1 & 0 \\
0 & 1 & 0 & 1 & 0 & 1 \\
0 & 0 & 1 & 0 & 1 & 1
\end{array}\right]
$$

MPA is an iterative process, where the soft information are exchanged between layer nodes and resource nodes. We define $\zeta_{j}=\left\{k \mid \boldsymbol{F}_{k j}=1, \forall k\right\}$ and $\xi_{k}=\left\{j \mid \boldsymbol{F}_{k j}=1, \forall j\right\}$, and the capacity of set $\zeta_{j}$ is $d_{c}$ for each cellular user $j$. At the $i$ th iteration, the extrinsic information from resource node $k$ to layer node $j$ is given by

$$
L_{k \rightarrow j}^{i}(x)=\sum_{\tilde{\boldsymbol{x}}: \tilde{x}_{j}=x}\left\{M_{k}(\tilde{\boldsymbol{x}}) \prod_{\tilde{j} \in \xi_{k} \backslash j} L_{\tilde{j} \rightarrow k}^{i-1}\left(\tilde{x}_{\tilde{j}}\right)\right\},
$$

where $M_{k}(x)$ means the computation of $M(x)$ for the $k$ th resource node [4]. Besides, the exchanged information from layer node $j$ to resource node $k$ is given by

$$
L_{j \rightarrow k}^{i}(x)=\prod_{\tilde{k} \in \zeta_{j} \backslash k} L_{\tilde{k} \rightarrow j}^{i}(x) .
$$

For the iterative computation, the initial value is $L_{j \rightarrow k}^{0}(x)=$ $1 / M$. When $I_{T}$ iterations is reached, we can get the hard estimation by

$$
\hat{\boldsymbol{x}}_{j}=\arg \max _{\hat{\boldsymbol{x}}}\left\{\prod_{k \in \zeta_{j}} L_{k \rightarrow j}^{I_{T}}(\boldsymbol{x})\right\} .
$$

\section{B. Low Complexity Decoding Algorithm for Cellular Users}

In MPA process, we need a large number of exponential operations to obtain (6), which brings high computational complexity. In our network, we need even more exponential operations and multiplications. For the implementation of hardware, we need appropriate method to reduce the complexity for the decoder of cellular users. LSD is proved to be an efficient method for general SCMA system, as only signals within a given hypersphere are considered instead of exhaustive search [14].

The design of general SCMA codebook is introduced in literature [15]. The process can help us to determine the relationship between non-zero SCMA codewords and equivalent lattice points. Let non-zero SCMA codeword $\boldsymbol{x}$ be $N$ dimensional for user $j$, and $\boldsymbol{u}_{2 N, j}$ be the equivalent $2 N$ dimensional QAM constellation. The codeword formula can be written as

$$
\boldsymbol{x}_{N, j}=\Delta_{j} \cdot\left(\boldsymbol{E}_{r}+i \cdot \boldsymbol{E}_{i}\right) \cdot \boldsymbol{M} \cdot \boldsymbol{u}_{2 N, j},
$$

where $\Delta_{j}$ denotes the constellation operators for user $j$, e.g., phase rotation, $\boldsymbol{M}$ denotes the unitary rotation matrix, $\boldsymbol{E}_{r}$ and $\boldsymbol{E}_{i}$ are the matrices which can select components from vector $\boldsymbol{u}_{2 N, j}$ that corresponds to the real part and imaginary part of QAM symbols, respectively. For instance, for the case of $N=2$ and $M=4$, the best rotation matrix $M$ has been found in literature [16], and $\boldsymbol{E}_{r}=\boldsymbol{I}$ while $\boldsymbol{E}_{i}=\boldsymbol{O}$.

Let $\boldsymbol{G}_{j}=\Delta_{j} \cdot\left(\boldsymbol{E}_{r}+i \cdot \boldsymbol{E}_{i}\right) \cdot \boldsymbol{M}$. The received signal on subcarrier $k$ can be rewritten as

$$
\begin{aligned}
y_{k} & =\tilde{\boldsymbol{h}}_{k}^{T} \operatorname{diag}\left\{\boldsymbol{g}_{k, 1}^{T}, \ldots, \boldsymbol{g}_{k, J}^{T}\right\} \boldsymbol{u}_{k}+h_{k}^{\prime} x_{k}^{\prime}+n_{k} \\
& =\boldsymbol{w}_{k} \boldsymbol{u}_{k}+z_{k},
\end{aligned}
$$

where $\tilde{\boldsymbol{h}}_{k}=\left[h_{k, 1}, \ldots, h_{k, J}\right]^{T}$, row vector $\boldsymbol{g}_{k, j}^{T}$ corresponds to the $k$ th row of matrix $\boldsymbol{G}_{j}, \boldsymbol{u}_{k}=\left[\boldsymbol{u}_{2 N, 1}^{T}, \ldots, \boldsymbol{u}_{2 N, J}^{T}\right]^{T}$ and $z_{k}$ denotes the interference of D2D users and noise in subcarrier $k$. Actually, only $d_{c}$ cellular users will collide in the same subcarrier, so the set of lattice points can be modified. We assume that $\boldsymbol{w}_{k}$ is $1 \times L$ vector and $\boldsymbol{u}_{k}$ is $L \times 1$ vector.

We use ML criterion to estimate SCMA transmitted codewords as shown in (2)-(4), and for the modified formula in (10), the ML detector searches the optimum $\hat{\boldsymbol{u}}_{k}$ in a brute-force way. Notice that some signal points have tiny contributions to the iterative process. Thus, we can set a 
radius $C$ to control the search range. Let $\tilde{\boldsymbol{y}}_{k}=\left(\begin{array}{c}y_{k} \\ \mathbf{0}\end{array}\right)$ be a $(L+1) \times 1$ vector and $\tilde{\boldsymbol{W}}_{k}=\left(\begin{array}{c}\boldsymbol{w}_{k} \\ \boldsymbol{I}\end{array}\right)$ be a $(L+1) \times L$ matrix which is also a column full rank matrix. Considering the general sphere decoder, we have

$$
\left\|\tilde{\boldsymbol{y}}_{k}-\tilde{\boldsymbol{W}}_{k} \boldsymbol{u}_{k}\right\|^{2} \leq C \text {. }
$$

To implement the sphere decoder, we apply the QR factorization for $\tilde{\boldsymbol{W}}_{k}$

$$
\tilde{\boldsymbol{W}}_{k}=\left[\boldsymbol{Q}_{1}, \boldsymbol{Q}_{2}\right]\left[\begin{array}{c}
\boldsymbol{R} \\
\mathbf{0}
\end{array}\right],
$$

where the dimension of $\boldsymbol{Q}_{1}, \boldsymbol{Q}_{2}$, and $\boldsymbol{R}$ are $(L+1) \times L$, $(L+1) \times 1$ and $L \times L$, respectively. Now we can rewrite (11) as

$$
\left\|\boldsymbol{y}_{k}^{\prime}-\boldsymbol{R} \boldsymbol{u}_{k}\right\|^{2} \leq C^{\prime}
$$

where $\boldsymbol{y}_{k}^{\prime}=\boldsymbol{Q}_{1}^{\dagger} \tilde{\boldsymbol{y}}_{k}$ and $C^{\prime}=C-\left|\boldsymbol{Q}_{2}^{\dagger} \tilde{\boldsymbol{y}}_{k}\right|^{2}$. Note that matrix $\boldsymbol{R}$ is an upper triangular matrix. We can expand (13) as

$$
\sum_{i=1}^{L}\left|y_{i}^{\prime}-\sum_{j=i}^{L} r_{i j} u_{j}\right|^{2} \leq C^{\prime} \text {. }
$$

Then the sphere decoder can work in a backward recursive way. For the sake of simplification, we drop the detail of search process. Without sphere decoder, all $M^{d_{c}}$ points should be candidate points, while the list sphere decoder finds all candidate lattice points within the given radius $C$, and the size of this set $\Phi_{n}$ is $T_{L S D}$.

Consider the exchanged information of MPA in log domain without sphere decoder, we have

$$
L_{k \rightarrow j}^{i}(x)=* \max _{\tilde{\boldsymbol{x}}: \tilde{x}_{j}=x}\left\{\log \left(M_{k}(\tilde{\boldsymbol{x}})\right)+\sum_{\tilde{j} \in \xi_{k} \backslash j} L_{\tilde{j} \rightarrow k}^{i-1}\left(\tilde{x}_{\tilde{j}}\right)\right\},
$$

where $* \max$ operation is given by

$$
\begin{aligned}
* \max (a, b) & =\log \left(e^{a}+e^{b}\right) \\
& =\max (a, b)+\log \left(1+e^{-|a-b|}\right) .
\end{aligned}
$$

By adding the sphere decoder, (15) can be modified as

$$
L_{k \rightarrow j}^{i}(x)=* \max _{\tilde{\boldsymbol{x}} \in \Phi_{n}}\left\{\log \left(M_{k}(\tilde{\boldsymbol{x}})\right)+\sum_{\tilde{j} \in \xi_{k} \backslash j} L_{\tilde{j} \rightarrow k}^{i-1}\left(\tilde{x}_{\tilde{j}}\right)\right\} .
$$

When we use Max-log-MPA, we usually have the approximation that $* \max (a, b) \approx \max (a, b)$.

\section{Complexity Analysis}

First we consider the computational process of LSD. The computation of vector $\boldsymbol{y}_{k}^{\prime}$ and scalar $C^{\prime}$ require $\mathrm{QR}$ factorization, which needs complex multiplications and complex summations. We use the modified Gram-Schmidt algorithm [17] to achieve QR factorization and this part requires $2 L^{3}+2 L^{2}+L$ complex operations with $L=d_{c} \cdot \log _{2}(M)$. After that, we need to run the search process in a backward recursive way as shown in (14). The expected complexity [18] for this part is shown in (18),

$$
E(i)=\sum_{i=1}^{L}(2 i+7) P_{i}
$$

where $P_{i}$ is the average number of points inside an $i$-th layer.

Next we analyze the computational complexity of MPA decoding. For Max-log-MPA and LSD-MPA, the distinction is absolutely reflected in the exchanged information from resource nodes to layer nodes. The computation of (15) in each iteration requires $K d_{c} M^{d_{c}}\left(d_{c}+3\left(J_{d 2 d}\right)^{M_{d 2 d}}\right)$ complex multiplications and $K d_{c} M^{d_{c}}\left(2 d_{c}-3+2\left(J_{d 2 d}\right)^{M_{d 2 d}}\right)+K d_{c} M$ complex summations, where $M_{d 2 d}$ is the codebook size of D2D users. However, the computation of (17) in each iteration requires $K d_{c} T_{L S D}\left(d_{c}+3\left(J_{d 2 d}\right)^{M_{d 2 d}}\right)$ complex multiplications and $K d_{c} T_{L S D}\left(2 d_{c}-2+2\left(J_{d 2 d}\right)^{M_{d 2 d}}\right)$ complex summations, where $T_{L S D}$ can be much lower than $M^{d_{c}}$. For the process of (7) and (8) in MPA decoding, the complexity remains unchanged with sphere decoding.

\section{Receiver for D2D Users}

For D2D communication, each D2D pair is allocated with one of the $K$ OFDMA tones. Regardless of the power diversity and distance diversity, let the $i$ th OFDMA tone be used by the $i$ th D2D pair $\left(i=1,2, \ldots, J_{d 2 d}\right)$. In the subcarrier $i$,

$$
y_{i}=h_{i, d} \cdot x_{i, d}+\sum_{j \in \xi_{i}} h_{j, c} \cdot x_{j, c}+n_{i} .
$$

We use subscript $d$ and $c$ to distinguish the D2D users from cellular users. Consider the interference of $d_{c}$ cellular users, the ML receiver is given by

$$
\hat{x}_{i, d}=\arg \max _{x \subset X_{d 2 d}} N_{i}(x),
$$

where

$$
N_{i}(x) \propto \sum_{\boldsymbol{t} \subset X_{c}} p\left(\boldsymbol{x}_{c}=\boldsymbol{t}\right) \cdot \exp \{G(\boldsymbol{t}, x)\} .
$$

Notice that the sphere decoder will change the search space for the interference of cellular users. For the D2D receiver in each D2D pair, the set of total SCMA transmitted codewords $X_{c}$ can be replaced by the set $\Phi_{i}$. Thus, we can modify (20) as

$$
N_{i}(x) \propto \sum_{\boldsymbol{t} \subset \Phi_{i}} p\left(\boldsymbol{x}_{c}=\boldsymbol{t}\right) \cdot \exp \{G(\boldsymbol{t}, x)\},
$$

where

$$
G(\boldsymbol{t}, x)=-\frac{1}{\sigma_{n}^{2}}\left\|\left(y_{i}-\boldsymbol{h}_{i, c}^{T} \boldsymbol{t}\right)-h_{i, d} x\right\|^{2} .
$$

For each D2D receiver, we need to compute $M^{d_{c}} M_{d 2 d}$ Euclidean distances in (21) without considering the sphere decoding, while for the computation of (22) we will compute $T_{L S D} M_{d 2 d}$ Euclidian distances. However, the process of LSD in each D2D receiver brings extra complex multiplications and complex summations. In general, The exponential operator is reduced by the proportion of $\frac{T_{L S D}}{M^{d_{c}}}$ which is more significant for the implementation of hardware. 


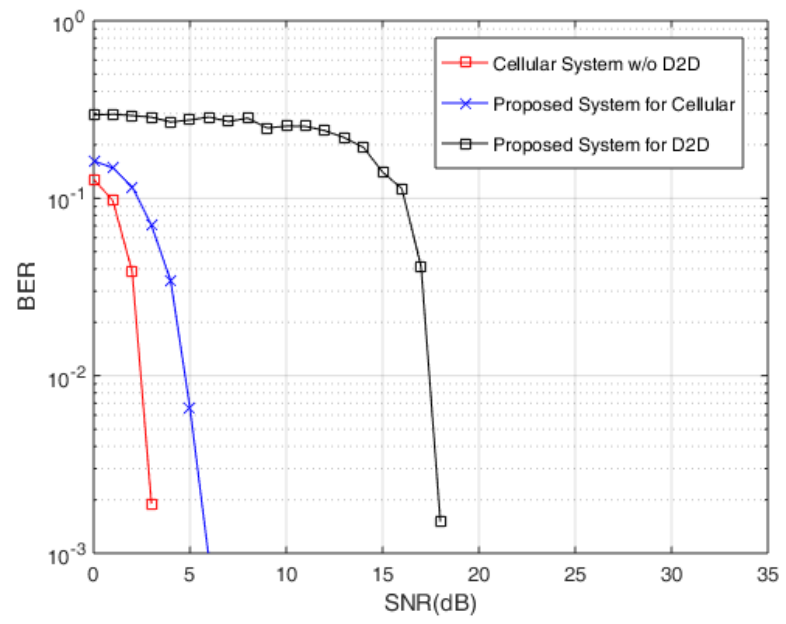

Fig. 3: BER performance of different system models.

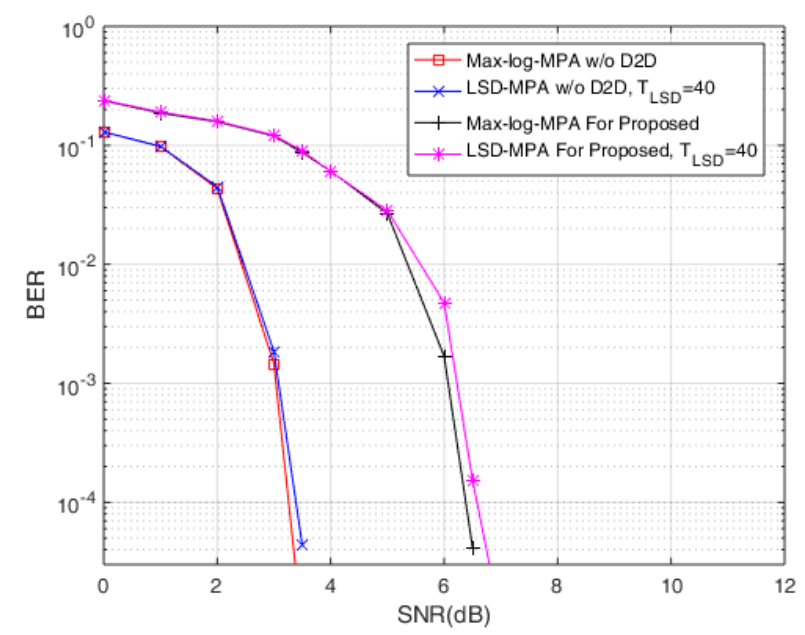

Fig. 4: BER performance of cellular users with AWGN channel.

\section{Simulation Results}

In this section, we perform simulation to demonstrate the BER performance of the proposed hybrid network with D2D users and uplink cellular users. We consider a system model where $J=6, K=4, N=2, M=4$, and $J_{d 2 d}=2$. The factor graph and the indicator matrix $F$ is defined in Fig. 2 and (5), respectively. We consider the case of single antenna. The codewords for D2D communication is modulated by BPSK. We employ two types of channel models, one is AWGN channel and the other is ideal Rayleigh fading channel. The channel code is set to $1 / 3$ turbo code for both cellular users and D2D users. Besides, the maximum number of MPA iteration $I_{T}$ is set to 6 .

\section{A. AWGN Channel}

In Fig. 3, we show the BER performance of the communication for both cellular users and D2D users, where the receiver is based on conventional MPA and ML criterion, respectively.

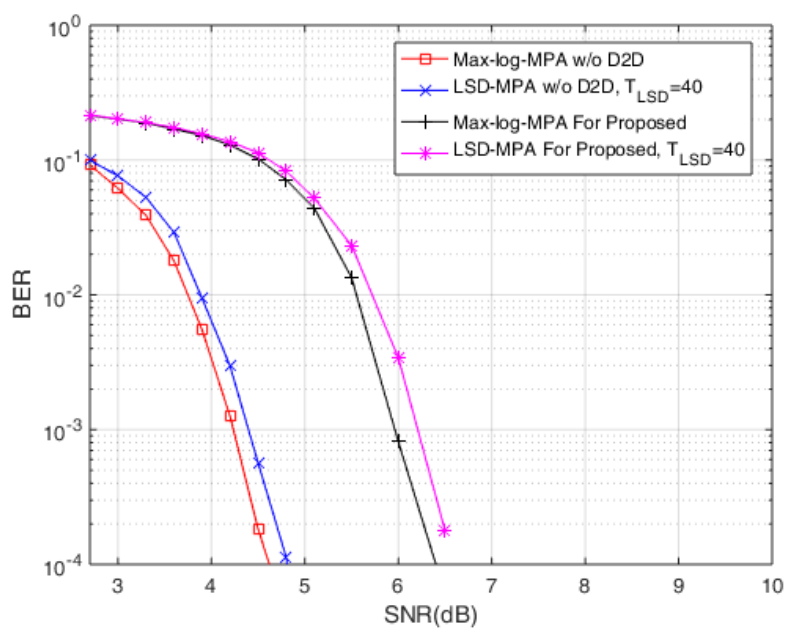

Fig. 5: BER performance of cellular users with Rayleigh fading channel.

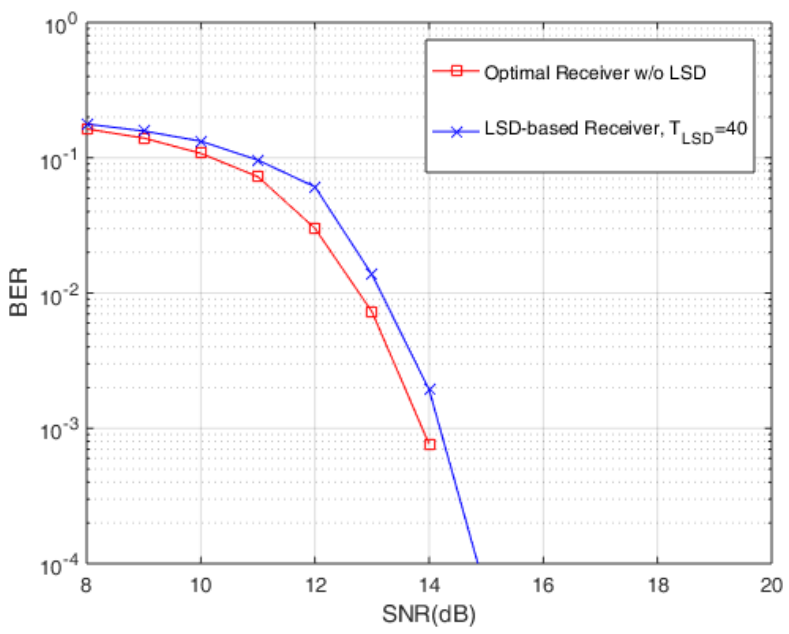

Fig. 6: BER performance of D2D users with Rayleigh fading channel.

From Fig. 3 we can observe that the BER performance of cellular users in the proposed network model has the loss of near $3 \mathrm{~dB}$ compared with general uplink SCMA system, which indicates the interference level from D2D users. Besides, the performance of D2D users is also degraded. The possible reason is that power diversity and distance diversity are not considered in this system.

Fig. 4 compares the BER performance of Max-log-MPA and LSD-MPA for both general SCMA receiver and the proposed SCMA receiver. For traditional MPA, since $M=4$ and $d_{c}=$ 3 , we can discover that $4^{3}=64$ Euclidean distances need to be computed. When we set $T_{L S D}=40$ in LSD based MPA, the search space is reduced. We can observe from Fig. 4 for general cellular network without D2D, the performance loss is near $0.3 \mathrm{~dB}$, which is negligible. For the proposed network, when BER equals to $1 \times 10^{-4}$, the performance loss is within $0.5 \mathrm{~dB}$, which is also negligible. 


\section{B. Ideal Rayleigh Fading Channel}

We focus on the performance of cellular users in Fig. 5. We still set $T_{L S D}=40$ in LSD based MPA to reduce the search space. From Fig. 5 we can observe that considering the channel is totally known for the receiver, the BER performance gap between general cellular network and proposed network is still near $3 \mathrm{~dB}$. Besides, compared to Max-log-MPA, the LSD-MPA only results in a performance loss of near $0.5 \mathrm{~dB}$.

In Rayleigh fading channel, we also compare the BER performance of D2D users which is shown in Fig. 6. We can observe that the LSD-based D2D receiver still results in negligible performance loss while the decoding complexity is reduced.

\section{Decoding Time Comparison}

Fig. 7 illustrates the comparison of decoding time in matlab platform between Max-log-MPA and LSD-MPA considering the process of D2D decoding. The SNR is set to $4 \mathrm{~dB}$ for both AWGN channel and Rayleigh channel, while other parameters remain unchanged. We can observe that when we set the appropriate $T_{L S D}$ as in Fig. 7, although some additive procedures are required in sphere decoding algorithm, the complexity is still reduced compared with traditional MPA. Therefore, this method is appropriate for our proposed system coexisting with D2D users.

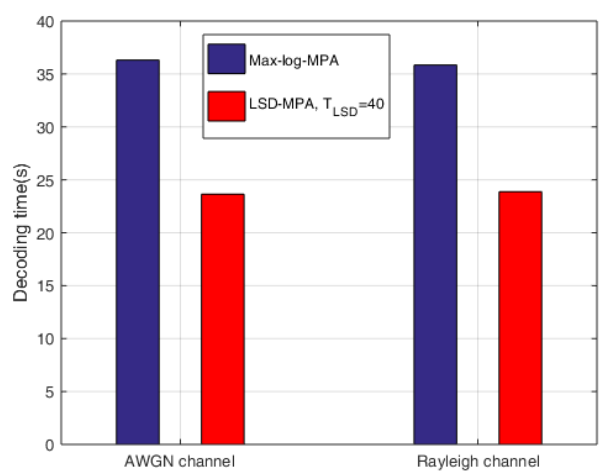

Fig. 7: Decoding time of different decoding algorithms.

\section{CONCLUSION}

In this paper, a D2D and uplink cellular coexisting network model is considered so that the overloading factor is larger than general SCMA system without D2D users. For the receiver design of the proposed system, conventional MPA brings a high complexity, so we adopt LSD-MPA to reduce the complexity, which also reduces the complexity of D2D receiver. Simulation results show that the BER performance for cellular users with LSD-MPA has negligible loss. Besides, in future work we hope to consider transmit power optimization and observe the performance of D2D communication.

\section{ACKNOWLEDGMENT}

This paper is supported by National Natural Science Foundation of China under Grant 61671294, STCSM Key Fundamental Project under Grant 16JC1402900 and 17510740700,
National Science and Technology Major Project under Grant 2018ZX03001009-002.

\section{REFERENCES}

[1] "5G: A technology vision," Huawei Technol. Co.. Ltd., Shenzhen, China, White Paper, Nov. 2013. [Online]. Available: www.huawei.com/ilink/en/download/HW_314849.

[2] M. N. Tehrani, M. Uysal and H. Yanikomeroglu, "Device-to-Device communication in 5G cellular networks: Challenges, solutions, and future directions," IEEE Commun. Mag., vol. 52, no. 5, pp. 86-92, May 2014.

[3] H. Nikopour and H. Baligh, "Sparse code multiple access," in Proc. IEEE 24th Int. Symp. Pers. Indoor Mobile Radio Commun. (PIMRC), Sep. 2013, pp. 332-336.

[4] H. Mu, Z. Ma, M. Alhaji, P. Fan and D. Chen, "A fixed low complexity message pass algorithm detector for up-link SCMA system," IEEE Wireless Commun. Lett., vol. 4, no. 6, pp. 585-588, Dec. 2015.

[5] Y. Du, B. Dong, Z. Chen and X. Wang, "A fast convergence multiuser detection scheme for uplink SCMA systems," IEEE Wireless Commun. Lett., vol. 5, no. 4, pp. 388-391, Aug. 2016.

[6] A. Bayestsh, H. Nikopour, M. Taherzadeh and H. Baligh, "Low complexity techniques for SCMA detection," in Proc. IEEE Globecom. Workshops. (GC Wkshps), Dec. 2015, pp. 1-6.

[7] Y. Liu, X. Fang, H. Yang and et al, "A mapping scheme of users to SCMA layers for D2D communication," in Proc. IEEE Veh. Technol. Conf. (VTC Spring), May 2016, pp. 1-5.

[8] K. Zhao, Y. Shi, Y. Dai and et al, "Resource allocation in Device-toDevice communication underlaid cellular network using SCMA: An opportunistic approach," in Proc. IEEE Int. Conf. Commun. China (ICCC), Shenzhen, China, Nov. 2015, pp. 1-6.

[9] Y. Dai, M. Sheng, K. Zhao and et al, "Interference-aware resource allocation for D2D underlaid cellular network using SCMA: A hypergraph approach," in Proc. IEEE Wireless Commun. Network. Conf. (WCNC), Apr. 2016, pp. 1-6.

[10] Y. Li, X. Sha, F. Jiang and L. Ye, "Sparse codes allocation in D2D communications underlaying cellular networks," in Proc. IEEE Int. Wireless Commun. Mobile Comput. Conf. (IWCMC), Sep. 2016, pp. 1-5.

[11] K. Zhao, M. Sheng, J. Liu and et al, "Graph-based joint mode selection and resource allocation scheme for D2D and cellular hybrid network using SCMA," in Proc. IEEE Wireless Commun. Signal Process. (WCSP), Oct. 2016, pp. 1-5.

[12] Y. Li, M. Sheng, Y. Zhu, T. Jiang and J. Li, "Sum rate maximization in underlay SCMA Device-to-Device networks," in Proc. IEEE Global Commun. (GLOBECOM), Washington, DC, USA, Dec. 2016, pp. 1-6.

[13] Z. Sun, M. Sheng, D. Zhai, Y. Zhang and J. Li, "Resource management for D2D underlaying cellular network with hybrid multiple access technologies," in Proc. IEEE Wireless Commun. Signal Process. (WCSP), Oct. 2016, pp. 1-5.

[14] F. Wei and W. Chen, "Low complexity iterative receiver design for sparse code multiple access," IEEE Trans. Commun., vol. 65, no. 2, pp. 621-634, Feb. 2017.

[15] M. Taherzadeh, H. Nikopour, A. Bayesteh and H. Baligh, "SCMA codebook design," in Proc. IEEE Veh. Technol. Conf. (VTC Fall), Sep. 2014, pp. 1-5.

[16] J. Boutros and E. Viterbo, "Signal space diversity: A power- and bandwidth-efficient diversity technique for the Rayleigh fading channel," IEEE Trans. Inf. Theory, vol. 44, no. 4, pp. 1453-1467, Jul. 1998.

[17] G. H. Golub and C. Van Loan, Matrix Computations. Baltimore, MD, USA: The John Hopkins Univ. Press, 1993.

[18] B. Hassibi and H. Vikalo, "On the sphere-decoding algorithm I. Expected complexity," IEEE Trans. Signal Process., vol. 53, no. 8, pp. 28062818, Aug. 2005.

[19] D. Zhai, M. Sheng, X. Wang and et al, "Energy-Saving resource management for D2D and cellular coexisting networks enhanced by hybrid multiple access technologies," IEEE Trans. Wireless Commun., vol. 16, no. 4, pp. 2678-2692, Apr. 2017.

[20] J. Liu, M. Sheng, L. Liu, Y. Shi and J. Li, "Modeling and analysis of SCMA enhanced D2D and cellular hybrid network," IEEE Trans. Commun., vol. 65, no. 1, pp. 173-185, Jan. 2017. 